Full citation: MacDonell, S.G., \& Fletcher, T. (1998) Metric selection for effort assessment in multimedia systems development, in Proceedings of the Fifth International Symposium on Software Metrics (Metrics'98). Bethesda MD, USA, IEEE Computer Society Press, pp.97-100.

doi: 10.1109/METRIC.1998.731232

\title{
Metric Selection for Effort Assessment in Multimedia Systems Development
}

\author{
Stephen G. MacDonell and Tim Fletcher \\ Department of Information Science \\ University of Otago, P O Box 56, Dunedin, New Zealand \\ email: stevemac@infoscience.otago.ac.nz,tfletcher@sol.otago.ac.nz
}

\begin{abstract}
This paper describes ongoing research directed at formulating a set of appropriate metrics for assessing effort requirements for multimedia systems development. An exploratory investigation of the factors that are considered by industry to be influential in determining development effort is presented. This work incorporates the use of a GQM framework to assist the metric selection process from a literature basis, followed by an industry questionnaire. The results provide some useful insights into contemporary project management practices in relation to multimedia systems.
\end{abstract}

\section{INTRODUCTION}

With advances in hardware and software technology, the feasibility of widespread multimedia use has reached a stage where all system developments can (and perhaps should) incorporate a variety of media components. In transaction-oriented systems, however, this generally extends only to the inclusion of graphics e.g. pictures of employees. As a result there remains a class of systems that is considered to be 'multimedia', used widely in education, entertainment and 'infotainment' (the interaction of the two prior domains). These systems generally incorporate some combination of sounds, animations, video clips, virtual reality and/or high quality graphics that, when effectively integrated, provide a semantically rich information environment. Whereas substantial research effort has been expended in measuring various aspects of traditional transaction processing and process control systems, little work has been performed in determining and evaluating measures for project management in relation to multimedia systems.

The question may well be asked - is there a need for yet more measures? In our view there are a number of reasons why new metrics are required:

- A fundamentally different software process. One of the most significant phases in multimedia systems development is content (or media) creation. Later in the process, all of the media components must be integrated, or 'authored', into a coherent system. There may be a need for storyboarding, to specify content and navigation. These tasks have no directly corresponding tasks in the development of transaction-centred or process control systems. Models available from these domains are therefore insufficient to take into consideration these other process steps.

- Distinct tools and techniques. Specialised contentoriented equipment and high-level visual authoring environments are widely employed in the creation and composition of multimedia systems, creating a different effort pattern than that found in development where 'standard' programming tools only are used, and about which we know much more.

- Different project management emphasis. Personnel responsible for managing software projects in the transaction-processing domain have often themselves come through developer ranks. As a result they may be familiar with methods like COCOMO [1] in terms of determining development effort. Multimedia project managers, however, are likely to come from a more diverse background from graphic design, television or film production. The models and assumptions that they bring to the task of project management may be substantially different and potentially more effective.

- Cross-disciplinary project teams. The personnel who make up multimedia development project teams may also have substantially different skills and backgrounds, more so than in teams brought together to construct traditional business or scientific systems [2]. Managing such a diverse team may be even more difficult than managing a more 'traditional' group of analysts and programmers; as part of this management process, predicting, coordinating, monitoring and controlling the team's development effort may be equally more difficult. 
It is asserted here that, given the conditions just stated, the direct application of existing models and measures is unlikely to meet the management needs for effort assessment and prediction in the multimedia systems domain. A new approach would seem to be warranted.

\section{COMPREHENSIVE ASSESSMENT OF FACTORS}

\subsection{Framework development}

In an attempt to select appropriate measures in a structured and rational way, the goal/question/metric (GQM) approach [3] was used. This is a decomposition-based method whose goal is to produce a hierarchy of measurement program levels. At the top, the program's goal is clearly stated. Questions are then asked that, when answered, will enable the goal to be achieved. Measures that are both sufficient and necessary to enable the questions to be answered are then specified at the lowest level of the hierarchy. Our goal is to determine system and component characteristics that are considered by industry to be influential in affecting multimedia systems development effort. Given that this goal is largely exploratory, questions have been included (mainly from the literature) that enable the consideration of both empirical and anecdotal factors within the framework. The questions asked have come from several sources:

- texts on multimedia project management (e.g. [2])

- texts on film and video project management (e.g. [4])

- comments on important considerations from informal phone calls made to developers

- software metrics texts in which system size tends to be the dominant factor (e.g. [1]).

Thus a wide variety of factors have been incorporated into the overall framework, in order to make the approach as comprehensive as possible. As data is collected the framework will be refined to only include factors that are indeed found to have some significant impact on development effort. Some of the issues currently considered in the framework are now discussed.

Development tool issues - Generally the nature of a project will determine which development tool or environment is used. Whilst authoring tools are the most common, other tools are also used, particularly where very complex processing is required. In these cases visual 3GLs, 'traditional' 3GLs, or a 3GL/authoring tool combination is often employed. Authoring tools generally offer visually based high productivity development under an eventdriven development model. As such these environments are either codeless or utilise a very high-level scripting language. In addition, authoring tools provide features useful to multimedia developers such as media databases, built-in system optimisation tools and intelligent palette handling. Features of this nature can play a significant part in terms of development effort.

Delivery platform issues - As a rule the (often clientimposed) choice of target platform dictates the media format and optimisation required. For example, all content developed for use on CD-I must conform to either PAL or NTSC standard screen specifications, since CD-I systems use a television as the display unit. The issue of file formats is also important in terms of the need for conversion and the effort required for this task. Any analog format such as S-VHS video or DAT audio must be converted to digital format in order to be usable.

Content development issues - Media content development is generally held to be the most labour-intensive part of multimedia systems development. Production effort may vary according to the media mix (e.g. whether there is extensive use of the more complex media forms of video and sound), the number of media components and the 'complexity' of those components. The impact of each media type on overall content development effort therefore needs to be assessed. If the media component does not already exist, it must be created and this obviously adds to development effort. If a component needs to be constructed then its complexity will have a bearing on the associated construction effort - what actually determines media component complexity is, however, difficult to say. In a small-scale preliminary empirical study [5], screen complexity was said to be a function of the number of components and links it contained. How do we measure the complexity of a graphic, however? We are currently investigating graphics and visualisation research in order to assist us with such a question. Assessment difficulties may be further exacerbated by the fact that artistic judgement plays a significant role in the decision as to when a component is satisfactorily completed. The desire for aesthetic quality may mean that accurate prediction of development effort is made all the more difficult.

Organisational capability issues - Organisational factors are unlikely to directly affect development of individual projects but they will influence the general manner in which systems are developed. At a fundamental level the size of an organisation (in terms of personnel) may be of some interest. The breakdown of personnel across functional areas may also indicate a pattern of effort requirement. The media development technology employed by the organisation is also likely to have a significant impact on the ease with which they are able to effectively build multimedia systems.

Personnel issues - As successful multimedia development requires staff with a wide variety of skills, personnel often come from a very diverse background. It may be useful to know the background of staff since this may affect how they develop systems, plan, cost and estimate projects. England and Finney [2] state that the core members of a multimedia system development team are a producer, one or more programmers and one or more graphic artists. An 
extended team may consist of various other specialists: video and sound personnel, administrative support, script writers, instructional designers and subject matter experts. The effort of all of these personnel must be considered in any model that attempts to be comprehensive.

\subsection{Pilot study}

In order to perform some preliminary verification of the framework, a small pilot study was undertaken. Structured interviews were conducted with three interactive multimedia development organisations. In terms of recording development effort data, only one of the three organisations made use of standard timesheets. In this case they were then able to employ a very simple effort estimation model for a system including $n$ screens: ( $(n)$ screens) $x$ (mean screen creation time). In the other two organisations effort estimation was performed based only on project managers' experience. Two of the three organisations built heavily graphical systems and, in their view, content creation was the major activity within development, and content specialists were the dominant personnel in their teams. Within the three organisations few personnel came from a computing background indeed the common backgrounds were in film or music.

Results also suggested that the type of project almost entirely determined the development environment and the workload among various development areas. Two of the three organisations built reasonably simple (in terms of programming and functionality) content-centred systems delivered on kiosks, CD-ROMs and the Internet, primarily using authoring tools. One organisation built more complex systems requiring custom-written data acquisition and processing modules. This organisation used a visual 3GL environment because it provided rapid application development features with a powerful language. Only this organisation found programming to be their major development activity. Component reuse was practised extensively by all three organisations.

\subsection{Industry Questionnaire}

Results of the pilot study were combined with the components of the GQM framework to enable the development of a postal survey instrument. Again, the focus was on determining the factors that significantly affect development effort for multimedia systems.

At the time of writing we had received valid responses from 22 multimedia systems developers. Whilst this is not a large number from which to draw conclusions, some trends are already evident. These are now discussed.

Personnel in the respondents' organisations are drawn from a variety of backgrounds. Most common were those with a graphics design or arts background, employed at sixteen of the 22 organisations. Thirteen organisations employed personnel with a computing background, five employed those with television/film experience, and four employed personnel with music or audio backgrounds.

Just ten of the 22 respondents confirmed that they used some form of formal methodology in their development of multimedia. Of these, four mentioned the use of storyboards and/or paper drafts, and five described the incorporation of a content acquisition/development phase. Only one reported the use of a typical business-oriented life cycle type process. In relation to the tracking of personnel effort, timesheets were used by fifteen of the respondents, most recording effort expended on development tasks at the system level. A small number (five) recorded the screen and/or component name being worked on, and eight recorded the module name.

The survey recipients were then asked to specify the techniques they used to estimate system development effort. Nineteen answered that experience of similar past projects was used, the number of system screens was used by eight of the respondents, the amount of digital video was employed as a driver of effort by seven organisations, five respondents used a ratio of development time to delivery time and just one used a cost per digital minute measure. Of the five who claimed use of a development time to delivery time ratio, only two supplied the ratio employed: 1000:1 and 120:1. Two respondents remarked that they were still coming to terms with estimation.

Those who cited the use of experience of similar past projects in estimation were then asked what made projects similar. Fifteen of the group agreed that both project size and type were important, fourteen of the 22 suggested that a similar mix of media elements was influential, and eight considered that a common target delivery platform had an impact on project similarity.

Component reuse was practised in an ad hoc sense by eighteen of the 22 respondents, although the use of a formally managed approach to reuse was not widespread (five sites). More importantly for this discussion, only thirteen of the sites made any adjustment to their effort estimates as a result of planned reuse. When asked to specify how this adjustment was performed, respondents provided a variety of answers describing 'unofficial' changes - it is clear that there was no systematic process in place for adjustment for reuse in any of the sites.

Twenty of the respondents confirmed that the selected development environment had a significant impact on development effort, largely in relation to familiarity with tools and methods. Recipients were then asked to rank from 1 to 9 a list of factors in terms of how much they influenced development effort. The results, as shown in Table 1, indicate that project size and experience of personnel rank equal first, while testing requirements and platform optimisation rank lowest of the factors provided.

Eight factors considered to be associated with the difficulty of multimedia authoring and/or programming were listed and respondents were asked to rank these. Once more, the 
experience of staff rated most significantly, and platformrelated issues least influential.

Table 1: Ranking of factors that influence multimedia systems development effort

\begin{tabular}{||l||c|c||}
\hline \hline Factor & $\begin{array}{c}\text { Mean } \\
\text { rank }\end{array}$ & $\begin{array}{c}\text { Median } \\
\text { rank }\end{array}$ \\
\hline \hline Experience of personnel & 2.7 & 2.0 \\
\hline Size of project & 2.7 & 2.0 \\
\hline Amount of original media & 3.3 & 3.0 \\
\hline Programming complexity & 3.7 & 3.0 \\
\hline Client's expected quality & 5.1 & 5.0 \\
\hline Need for outside services & 6.4 & 6.0 \\
\hline Choice of platform & 6.3 & 6.5 \\
\hline Testing requirements & 7.0 & 7.0 \\
\hline Platform optimisation & 8.5 & 9.0 \\
\hline \hline
\end{tabular}

Table 2: Ranking of factors that influence authoring or programming difficulty

\begin{tabular}{||l|c|c||}
\hline Factor & $\begin{array}{c}\text { Mean } \\
\text { rank }\end{array}$ & $\begin{array}{c}\text { Median } \\
\text { rank }\end{array}$ \\
\hline \hline Experience of staff & 3.1 & 2.0 \\
\hline Type of tool used & 3.5 & 3.0 \\
\hline Need for a custom solution & 3.8 & 3.0 \\
\hline Special project requirements & 3.8 & 3.0 \\
\hline Need for database handling & 4.3 & 5.0 \\
\hline Other platform requirements & 4.4 & 4.0 \\
\hline Need for a search engine & 5.4 & 6.0 \\
\hline Platform optimisation needs & 7.0 & 7.5 \\
\hline
\end{tabular}

When asked how their background helped them estimate project costs, one (fortunate) respondent cited a 'natural ability' for planning and estimation. More common answers included the application of prior experience, the use of a "hit and miss" strategy, a sense that no two projects are the same so estimation based on history is immaterial, and the broader feeling that background hadn't helped at all - "this is a totally new environment and costings have to be developed from scratch"; "very little estimation, costs tend to be high". One respondent in fact remarked that his/her background made estimation more difficult. A more general question on the difficulties of project estimation elicited eight responses relating to a lack of realism in clients' perceptions of the ease of accommodating requirement changes and technology capability. Six respondents commented that the environments and tools used were so new that accurate estimation was not yet possible. Other difficulties mentioned were other unexpected changes (not related to client needs) and external constraints, such as having to wait for days without rain for filming.

\subsection{Discussion}

Whilst the results above are preliminary, some insights can be gained. The fact that nearly half the respondents indicated no use of a formal methodology may suggest that, in terms of maturity, they may be considered to be chaotic in their approach. When coupled with the widespread employment of personnel with non-computing backgrounds it is not entirely unexpected that product and process measurement has received only scant attention. In terms of methods used in estimation, none of the 'traditional' software metrics approaches (e.g. COCOMO, FPA) were mentioned; rather, the clearly favoured method is to use experience of previous projects, particularly in terms of size and type. Moreover, the tracking of effort is generally performed at a very high level. This again fits the model of relatively immature project management practices.

As in many development domains, staff experience and project size are perceived to be the most significant factors in determining development effort. The most influential media-related factor, the amount of original media, ranked third of the nine factors. Issues related to the delivery platform, thought to be potentially significant in our framework, appear to be relatively unimportant.

The general comments obtained from the respondents further reinforce the currently ad hoc approach of most to estimation. Although some are attempting to improve their practices there is still significant uncertainty as to how this can be achieved. Whilst the same may be said in regard to the management of other development domains, the extent of process immaturity in multimedia systems development seems to be greater.

\section{SUMMARY AND CONCLUSIONS}

This research is ongoing - it has produced only preliminary outcomes at this stage. We are encouraged by the industry verification of the framework in the pilot study and questionnaire. Further responses should provide greater insight into industry opinion on the factors that are related to multimedia systems development effort.

\section{REFERENCES}

[1] Boehm, B. (1981), Software Engineering Economics, Englewood Cliffs NJ, Prentice Hall

[2] England, E. and Finney, A. (1996), Managing Multimedia, Harlow, Addison-Wesley

[3] Basili, V.R. and Rombach, H.D. (1988), "The TAME Project: towards improvement-oriented software environments", IEEE Transactions on Software Engineering 14(6), 758-773

[4] Fraser, C. (1994) The Production Assistant's Survival Guide, BBC Television Training

[5] Fletcher, T., MacDonell, S.G. and Wong W.B.L. (1997), "Early experiences in measuring multimedia systems development effort" in Multimedia Technology and Applications (Proc. ICMTM '96), Hong Kong, Springer-Verlag, 211-220 